\title{
«Les médecins ne sont pas habitués à demander de l'aide»
}

\author{
Grâce à ReMed, les médecins en situation de crise peuvent demander et recevoir de \\ l'aide. Après une phase pilote positive, le réseau de soutien est désormais actif dans \\ toute la Suisse. Dans I'interview suivante, le Docteur Michael Peltenburg, co-initiant \\ et responsable du programme ReMed, explique comment ce réseau fonctionne et à \\ qui il s'adresse.
}

Interview:

Jacqueline Wettstein

Responsable de la communication FMH

Correspondance:

Dr Michael Peltenburg

Spécialiste en médecine

générale FMH

$\mathrm{CH}-8340$ Hinwil

michael.peltenburg@hin.ch

\section{Qu'est-ce que ReMed?}

Michael Peltenburg: En tant que médecins, nous exerçons un métier passionnant mais qui nous met fortement à contribution. Notre travail est exigeant, car il touche aux êtres humains et à leur vie. Lorsqu'un médecin connaît une crise personnelle, cela peut avoir des conséquences sur la qualité de son travail. C'est là que ReMed intervient en proposant un soutien: nous accompagnons les médecins concernés et leur montrons de nouvelles voies pour sortir de situations apparemment sans issue.

\section{Comment ReMed fonctionne-t-il?}

Le premier contact avec ReMed passe par l'un des quatre médecins du comité de direction. Le conseiller discute avec le médecin en situation de crise des démarches futures: pour quelle raison a-t-il pris contact? Quel type de soutien est attendu? ReMed est donc essentiellement un service d'accueil proposant une intervention de crise, un mentoring, un coaching et une évaluation. Selon la nature du problème, le conseiller indique des professionnels qualifiés - par exemple des psychiatres ou psychologues - ou des institutions spécifiques.

\section{A qui s'adresse ReMed?}

ReMed s'adresse aux médecins en situation de crise. L'entourage et les collaborateurs de médecins peuvent également prendre contact avec ReMed. ReMed réagit dans les 72 heures, voire plus rapidement en général. L'expérience montre que les médecins en situation de crise tardent souvent à s'adresser à nous. C'est pourquoi nous recommandons aux personnes concernées de contacter ReMed le plus tôt possible, avant que la crise n'atteigne son paroxysme.

Quelles sont les personnes qui consultent et quels problèmes ont-elles?

Les médecins s'adressent à nous avec des problèmes très variés. Il peut s'agir par exemple d'un conflit avec un supérieur hiérarchique ou d'un surmenage professionnel permanent. Bien évidemment, ReMed apporte

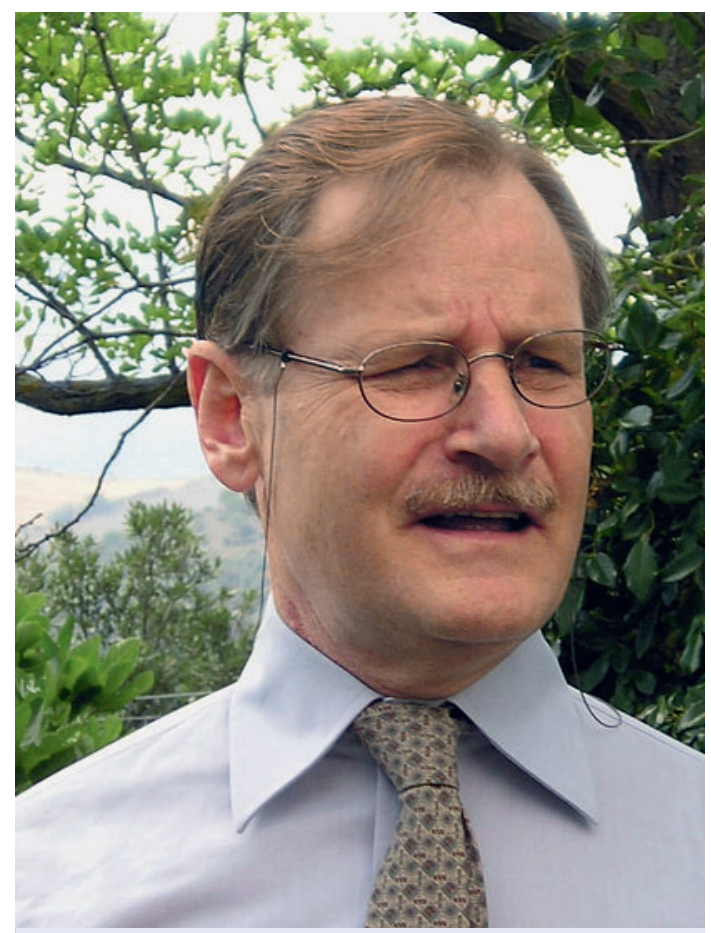

Dr Michael Peltenburg, co-initiant et responsable du programme ReMed.

également son soutien en cas de difficultés personnelles, comme des conflits continus à la maison ou un divorce.

\section{Que peut faire ReMed?}

Les médecins s'engagent au quotidien pour leurs patients. Mais lorsqu'il s'agit d'eux-mêmes, ils ne sont pas habitués à demander de l'aide. Ils ont souvent du mal à admettre qu'ils ont besoin d'un soutien. Avec ReMed, les médecins en situation de crise rencontrent des médecins, pour les conseiller. Ces derniers savent de quoi ils parlent et connaissent les difficultés d'après leur propre expérience. Nous souhaitons aider les médecins à améliorer leur santé et à la maintenir. Nous souhaitons également qu'ils continuent à exer- 
cer leur profession avec passion et à garantir ainsi des soins médicaux sûrs et de haute qualité à la population.

ReMed poursuit également un objectif de prévention - de quelle manière?

Le corps médical ne s'occupe pas assez de sa propre santé. Avec ReMed, nous voulons en faire prendre conscience à nos confrères: notre propre santé est essentielle et nous devons en prendre soin.

Qui se trouve derrière ReMed?

L'organe responsable est la FMH: lorsque nous avons présenté le projet au Comité central, les réactions ont immédiatement été positives. Nous menons ce projet en collaboration avec le Département Données, Démographie et Qualité DDQ de la FMH. Mais même si la FMH finance ReMed, elle n'a à aucun moment accès aux dossiers personnels et d'accompagnement. ReMed est lié au secret médical.

\section{La FMH n'a accès aux dossiers personnels et d'accompagnement à aucun moment}

La phase pilote est achevée et ReMed sera proposée dans toute la Suisse dès l'automne 2010. Quelles sont maintenant les prochaines étapes?

Depuis le lancement du projet en 2007, ReMed est intervenu activement près de 80 fois. Ceci montre que les médecins ont confiance en ReMed. Il s'agit désormais d'accroître la notoriété du réseau. ReMed va maintenant s'étendre officiellement à toute la Suisse. Nous avons prévu toute une série de mesures de communication telles que dépliants ou affiches pour les hôpitaux. Les médecins doivent savoir qu'ils peuvent s'adresser à ReMed dans les moments critiques.

\section{ReMed - pour les médecins en situation de crise}

ReMed conseille et accompagne les médecins en situation de crise. Il peut s'agir d'un conflit sur le lieu de travail ou d'une crise personnelle comme un divorce. Le réseau de soutien réagit dans les 72 heures après la prise de contact. ReMed accompagne ensuite les personnes concernées en leur montrant des possibilités d'action. ReMed est lié au secret médical.

\section{Contact}

Vous vous trouvez dans une situation critique et cherchez de l'aide? Ou vous connaissez un médecin de votre entourage qui a besoin d'un soutien? Contactez ReMed sur la ligne d'assistance disponible 24 h sur 24: 0800073633 ou sur help@swiss-remed.ch

\section{Informations}

Vous souhaitez en savoir davantage et sensibiliser vos collègues à l'action de ReMed? Vous pouvez commander gratuitement des dépliants et des affiches, en allemand et en français, à l'adresse suivante: info@swiss-remed.ch.

Vous trouverez de plus amples informations sur le site Internet www.swiss-remed.ch

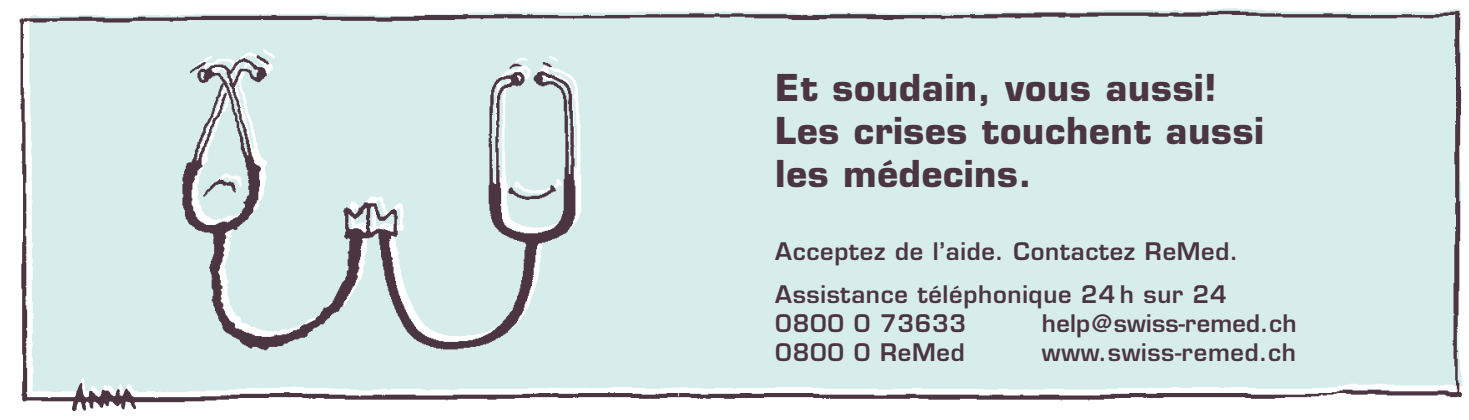

